\title{
Verbessert die Einnahme von Statinen die Überlebenschance?
}

Fragestellung: Die Einnahme von Statinen führt in der Sekundärprävention des Schlaganfalls möglicherweise zu einer erhöhten Rate von intrazerebralen Blutungen. Ob Statine die Prognose bei Patienten mit intrazerebralen Blutungen beeinflussen, ist bisher nicht bekannt.

Hintergrund: Statine sind hochwirksam in der Sekundärprävention des ischämischen Schlaganfalls. In der SPARCL-Studie zeigte sich allerdings bei Patienten, die $80 \mathrm{mg}$ Atorvastatin erhielten, ein erhöhtes Risiko intrazerebraler Blutungen verglichen mit Patienten, die Placebo erhielten $[1,2]$. Dieses Ergebnis konnte aber in einer großen Metaanalyse nicht repliziert werden [3].

Patienten, die einen ischämischen Insult erleiden, haben eine bessere Prognose, wenn sie zum Zeitpunkt der zerebralen Ischämie Statine einnehmen [4]. Die Prognose verbessert sich auch, wenn eine vorbestehende Statin-Medikation bei Patienten mit ischämischem Insult fortgeführt wird [5]. Ob es ähnliche Effekte bei Patienten mit zerebralen Blutungen gibt, war bisher nicht bekannt.

Patienten und Methodik: Bei der vorliegenden Studie handelt sich um eine retrospektive Analyse von 3.481 Patienten, die in einem Zeitraum von zehn Jahren mit intrazerebralen

Flint AC, Conell C, Rao VA et al. Effect of statin use during hospitalization for intracerebral hemorrhage on mortality and discharge disposition. JAMA Neurol. 2014; 71: $1364-71$
Blutungen in 20 verschiedenen Krankenhäusern in Nordkalifornien aufgenommen worden waren. Die Diagnose stützte sich auf die zerebrale Bildgebung. Bei allen Patienten wurden aus den
Krankenakten die wesentlichen prognostischen Faktoren gewonnen. Der primäre Endpunkt für diese Analyse war das Überleben nach 30 Tagen sowie die Frage, ob die Patienten nach Hause oder in eine stationäre Einrichtung verlegt wurden. Die Patienten wurden dahingehend unterteilt, ob sie vor oder während des Krankenhausaufenthaltes Statine einnahmen. Die statistischen Analysen wurden für die wesentlichen Komorbiditäten korrigiert.

Ergebnisse: Die Patienten waren im Mittel 73 Jahre alt. Bei $85 \%$ der Patienten war eine Hypertonie bekannt. Von den $3.481 \mathrm{~Pa}-$ tienten nahmen 1.194 Statine ein und 2.287 waren bezüglich Statinen unbehandelt. Die Patienten mit zerebralen Blutungen, die während des stationären Aufenthaltes ein Statin erhielten, hatten eine 30-Tage-Mortalität von 18,4\%, diejenigen, die kein Statin erhielten, hatten eine Sterblichkeit von 38,7\%. Dieser Unterschied war bei einer Odds Ratio von 4,25 statistisch signifikant.

Die Patienten, bei denen die Statine im Krankenhaus abgesetzt wurden, hatten eine Sterblichkeit von $57,8 \%$ verglichen mit $18,9 \%$ für Patienten bei denen eine vorbestehende Statin-Medikation fortgeführt wurde. Dieser Unterschied war mit einer Odds Ratio von 0,16 ebenfalls signifikant. Patienten, die Statine erhielten oder bei denen eine vorbestehende Statin-Therapie fortgesetzt wurde, wurden auch sehr viel häufiger nach Hause entlassen.

Schlussfolgerungen: Patienten mit zerebralen Blutungen, die mit Statinen vorbehandelt sind und bei denen die Statin-Medikation im Krankenhaus fortgeführt wird, haben eine deutlich bessere Überlebenschance als Patienten ohne Gebrauch von Statinen oder bei denen die Statine abgesetzt werden.

\section{- Kommentar von Hans-Christoph Diener, Essen}

\section{Fortsetzung der Statin-Therapie sollte prospektiv untersucht werden}

Die Ergebnisse dieser großen retrospektiven Studie aus den USA sind ermutigend, denn sie zeigen, dass definitiv kein erhöhtes Risiko besteht, wenn Patienten, die mit Statinen behandelt werden, eine zerebrale Blutung erleiden. Die Ergebnisse weisen sogar eher darauf hin, dass eine vorbestehende StatinMedikation im Falle einer zerebralen Blutung fortgeführt werden sollte. Ob Patienten mit zerebralen Blutungen von der Gabe von Statinen profitieren, wenn die Behandlung im Rahmen der zerebralen Blutung begonnen wird, ist allerdings bisher nicht bekannt.

Ein wesentlicher Nachteil ist, dass diese Studie retrospektiv erfolgte und nicht auf einer randomisierten Zuordnung der Patienten beruhte. In den statistischen Analysen waren die
Autoren allerdings bemüht, die wesentlichen Risikofaktoren und vaskulären Begleiterkrankungen zu korrigieren. Dessen ungeachtet wäre eine randomisierte Studie, welche die Frage untersucht, ob eine vorbestehende Statin-Therapie bei Patienten mit intrazerebralen Blutungen fortgeführt werden soll, sehr wünschenswert.

\footnotetext{
Referenzen:

1. Goldstein LB et al. Neurology 2007; Dec 12 [Epub ahead of print]

2. Amarenco P et al. Stroke 2007; 38: 3198-204

3. McKinney JS et al. Stroke 2012; 43: 2149-56

4. Flint AC et al. Stroke 2012; 43: 147-54

5. Blanco M et a. Neurology 2007; 69: $904-10$
} 